\title{
Total internal reflection from nematic liquid crystals
}

\author{
H. P. Hinov \\ Institute of Solid State Physics, Bulgarian Academy of Sciences, Sofia 1184, Bulgaria (*) \\ and Laboratoire de Physique des Solides, Bât. 510, Université de Paris-Sud, 91405 Orsay, France \\ and S. Sainov \\ Central Laboratory of Optical Storage and Information Processing, \\ Bulgarian Academy of Sciences, Sofia 1113, Bulgaria \\ (Reşu le 15 septembre 1978, révisé le 22 août 1979 et le 14 avril 1980, accepté le 16 avril 1980)
}

\begin{abstract}
Résumé. - Les conditions de la réflection totale atténuée (CRTA) sont obtenues pour une onde plane, monochromatique et homogène polarisée rectilignement et se réfléchissant sur un plan séparant deux milieux : un milieu isotrope - (prisme de flint-glass) et un milieu anisotrope - (cristal liquide nématique homéotrope déformé sous l'action d'une tension constante ou alternative à fréquence élevée).

Les résultats théoriques qui ont été obtenus avec la métho'de de WBK montrent deux conditions essentielles pour le comportement de l'onde : une condition concernant les variations exponentielles des champs électromagnétiques et la condition CRTA obtenue à partir de l'égalité entre les modules des coefficients incident et réfléchi. L'influence de la plaque de verre (c'est-à-dire la profondeur de pénétration de l'onde évanescente dans le cristal liquide) a été estimée. Les deux premiers termes de la condition de réflexion totale atténuée ont été utilisés dans une expérience réalisée avec des couches de MBBA. L'énergie d'ancrage du cristal liquide interagissant avec une couche très mince de lecithine a été déterminée approximativement autour de la valeur de $2 \times 10^{-2} \mathrm{erg} / \mathrm{cm}^{2}$. La précision de la méthode est faible pour les déformations comprises entre 0 et $5^{\circ}\left(\sim 1^{\circ}\right)$ et plus élevée pour les autres déformations.

Les mesures de la réflexion totale atténuée donnent une information nouvelle et importante sur la formation des couches électriques doubles, l'inhomogénéité du champ électrique et les propriétés flexoélectriques des cristaux liquides examinés.
\end{abstract}

\begin{abstract}
The attenuated total internal reflection (ATIR) conditions for a plane monochromatic wave, incident on the interface flint-glass prism-deformed homeotropic nematic layer with applied d.c. or high frequency a.c. electric field are considered.

The theoretical results obtained with the aid of WBK method show that there are two essential conditions for the wave behaviour : the condition for the exponential variations of the electromagnetic wave components and the ATIR condition obtained from the requirement for equality between the moduli of the incident and the reflected amplitudes. Estimates for the influence of the lower limiting glass plate (e.g. the penetration depth of the evanescent wave) are made. The first two terms in the ATIR condition were used in the experiment performed with MBBA films. The surface energy of the liquid crystal molecules interacting with a thin lecithin layer giving a homeotropic orientation was determined to be in the range of $2 \times 10^{-2} \mathrm{erg} / \mathrm{cm}^{2}$. The accuracy of the method is low in the range of deformation angles $0-5^{\circ}\left(\sim 1^{\circ}\right)$ and higher for the other regions.

The total internal reflection measurements give new and important information about the formation of the double electric layers, the inhomogeneity of the electric field and the flexoelectric properties of the investigated liquid crystals.
\end{abstract}

1. Introduction. - The surface behaviour of nematic liquid crystal layers is of great importance when considering technical applications and the exact and correct physical interpretations of all effects displayed in these materials. Many papers in recent years have been devoted to this problem. Much is done with respect to the physico-chemical preparation of the substrates in order to obtain strong or weak anchoring of the liquid crystal layers, homeotropic, tilt or planar orientation, etc...

Less is done unfortunately with regard to the determination of the exact deformation angle in the electrode surface regions. There are indeed sensitive

(*) Permanent address. optical methods permitting the solution of this problem. Many optical methods [1]-[5] were utilized for the measurement of the tilt bias angle (uniform tilt in the liquid crystal layer). T. Scheffer and J. Nehring [6] used a very sensitive magnetic null method making possible the determination of the tilt bias angle directly with only one measurement with an accuracy of $0.1^{\circ}$ regardless of the angle size or the nature of the nematic liquid crystal.

The attenuated total internal reflection (ATIR) as a useful method for measurement of the liquid crystal orientation has been used for the first time in 1934 by Freedericksz and Zwetkoff [7]. Selective and total reflections in cholesteric liquid crystals were utilized by many authors [8]-[11].

Stationary and modulated IR-ATIR spectra of 
Schiffs base nematic liquid crystals were reported by U. Fringeli et al. [12].

A. Hatta et al. [13], [14] measured infrared dichroism of MBBA using attenuated total internal reflection and determined the degree of the orientational order in the surface regions. ATIR was used for technical applications as well [15]-[16].

D. Rivière [17] applied the wave optics theory for ATIR from homogeneously oriented (planar, homeotropic, tilted) nematic liquid crystal layers and measured the refractive indices and the degree of orientation for the liquid crystal molecules of the nematic $6 \mathrm{CB}$. Rivière also discussed a number of important questions such as the accuracy of this method, the temperature variation of the refractive indices, the divergence of the laser beam etc... Rivière, Levy and Imbert [18], [19] determined with the aid of ATIR the refractive indices of the liquid crystal 6 CB with an accuracy of $10^{-3}$ and obtained the tilt angle for an obliquely oriented liquid crystal in the vicinity of the substrate. Rivière, Levy and Guyon [20] determined with the aid of ATIR the surface energy of a wedge shaped nematic liquid crystal $6 \mathrm{CB}$ with one substrate obliquely evaporated with two $\mathrm{SiO}$ films and the other substrate treated for a hotrieotropic orientation. Sh. Naemura [21] measured the anisotropic interfacial interactions between a nematic liquid crystal MBBA and various substrates in a magnetic field using a total internal reflection method as well.

The aim of this paper is a theoretical investigation of the propagation of a plane transverse magnetic (TM) plane wave in a deformed nonabsorbing homeotropic nematic liquid crystal in order to regard the possibilities for ATIR realization. The problem is solved using the solutions for the magnetic field component $H_{y}$. The differential equation for the amplitude of $H_{y}-\left|H_{y}\right|$ is transformed into a normal form for the component $\left|H_{y t}\right|$ and is solved for the case of WBK approximation. The electric field components $E_{x}$ and $E_{z}$ and the value of the reflectivity coefficient $V_{\mathrm{TM}}$ were calculated as well from the solution of the magnetic field component. The WBK ATIR conditions are obtained for this case and the influence of the lower surface is discussed.

It is shown that the formulae obtained in the references [16] and [17] for the homogeneously tilted layers may be used with a good accuracy and for thick weak deformed homeotropic nematic liquid crystals. The ATIR conditions were calculated for MBBA with the aid of the refractive indices given by M. Brunet-Germain [22] for various temperatures. The surface orientations in homeotropic deformed MBBA layers were determined in the performed experiment. The comparison between the surface orientation experimentally obtained and the theoretical results of J. Nehring et al. [23] allows in some cases the determination of the surface energy of the liquid crystal molecules. ATIR experimental results shed a new light on the flexoelectric gradient deformations [24]-[25] and on the formation of the electric double layers.

2. Theory. - The wave propagation in anisotropic absorbing and nonabsorbing films has been investigated extensively [26]-[27], etc. particularly in thin crystal and liquid crystal film optical waveguides [28][33]. The solution is based on the so-called $4 \times 4$ matrix method described by Billard [34], Smith [35], Teitler and Henvis [36], Berreman and Scheffer [37] and Berreman [38], [39] etc... This method was also applied by $\mathrm{T}$. Shimomura et al. [40] for measuring the electrooptical properties of nematic liquid crystal cells in obliquely incident light.

In this part of the paper the authors shall follow however the approach of $\mathrm{Ch}$. Hu et al. [32] which permits the obtaining of the main theoretical formulae applicable for ATIR measurements at appropriate extension.

Let us consider a plane wave obliquely incident on the interface $X O Y$ flint-glass - homeotropic nematic layer symmetrically deformed along $Z$ with negative low frequency dielectric anisotropy (Fig. 1) assuming simple time dependence exp $i \omega t$ and a polarization in the incident plane $X O Z$ (TM wave). The refractive index of the flint-glass is usually in the range of $N \sim 1.9$. The nematic liquid crystal is with a positive optical anisotropy having an ordinary index $n_{0}$ and an extraordinary index $n_{\mathrm{e}}>n_{0}$ - functions of the temperature and the frequency of the propagating wave.

The Maxwell equations with these assumptions are $(\mu=1$ everywhere) :

$$
\begin{aligned}
& \operatorname{rot} \mathbf{E}=-i(\omega / c) \mathbf{H} \\
& \operatorname{rot} \mathbf{H}=i \hat{\varepsilon}(\omega / c) \mathbf{E}
\end{aligned}
$$

where $\hat{\varepsilon}$ is the dielectric tensor of the nematic liquid crystal and has the form :

$$
\hat{\varepsilon}=\left[\begin{array}{ccc}
\varepsilon_{11} & 0 & \varepsilon_{13} \\
0 & \varepsilon_{22} & 0 \\
\varepsilon_{13} & 0 & \varepsilon_{33}
\end{array}\right]
$$

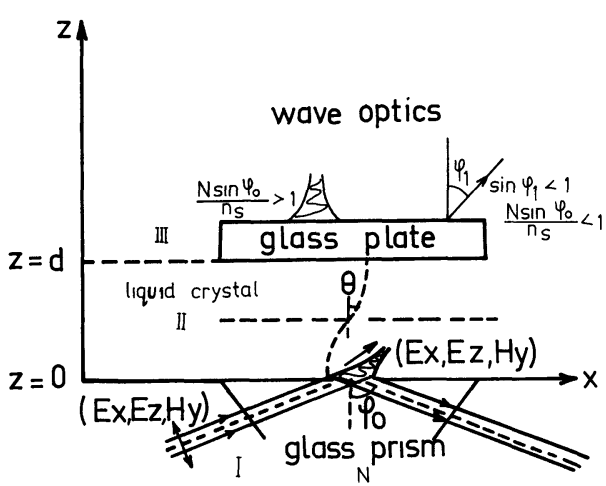

Fig. 1. - ATIR from interface glass-flint $60^{\circ}$ prism deformed initially homeotropic nematic liquid crystal : wave optics. 
where

$$
\begin{aligned}
& \varepsilon_{11}=n_{0}^{2} \cos ^{2} \theta+n_{\mathrm{e}}^{2} \sin ^{2} \theta \\
& \varepsilon_{22}=n_{0}^{2} \\
& \varepsilon_{33}=n_{0}^{2} \sin ^{2} \theta+n_{\mathrm{e}}^{2} \cos ^{2} \theta \\
& \varepsilon_{13}=\left(n_{\mathrm{e}}^{2}-n_{0}^{2}\right) \sin \theta \cos \theta .
\end{aligned}
$$

Ch. Hu and J. Whinnery [32] have obtained the equations describing the phase function $B(z)$ and the amplitude $\left|H_{y}\right|$ of the magnetic field component $H_{y}$ :

$$
H_{y}=\left|H_{y}\right| \exp i B(z) \exp i(\omega t-\beta x)
$$

(in our case $g_{33}, g_{11}, z$ and $x$ must be substituted for $g_{11}$, . $g_{33}, x$ and $z$ respectively) :

$$
\begin{gathered}
\frac{\mathrm{d}}{\mathrm{d} z} B=-\beta g_{13} / g_{11} \\
g_{11} \frac{\mathrm{d}^{2}\left|H_{y}\right|}{\mathrm{d} z^{2}}+\frac{\mathrm{d} g_{11}}{\mathrm{~d} z} \frac{\mathrm{d}\left|H_{y}\right|}{\mathrm{d} z}+ \\
+\left[k^{2}-\frac{\beta^{2}}{g_{11} n_{0}^{2} n_{\mathrm{e}}^{2}}\right]\left|H_{y}\right|=0
\end{gathered}
$$

where $k^{2}=\omega^{2} / c^{2}$

$$
\begin{aligned}
& g_{11}=\frac{\cos ^{2} \theta}{n_{0}^{2}}+\frac{\sin ^{2} \theta}{n_{\mathrm{e}}^{2}}, \\
& g_{13}=\left[\left(1 / n_{\mathrm{e}}^{2}\right)-\left(1 / n_{0}^{2}\right)\right] \sin \theta \cos \theta \\
& g_{33}=\frac{\sin ^{2} \theta}{n_{0}^{2}}+\frac{\cos ^{2} \theta}{n_{\mathrm{e}}^{2}}, \quad g_{22}=1 / n_{0}^{2} .
\end{aligned}
$$

The phase constant along $X \beta$ was determined from the boundary conditions. They require the equality of the phase factors of the magnetic field component at the flint-glass prism - the deformed homeotropic nematic layer interface :

$$
\beta^{2}=\frac{\omega^{2}}{c^{2}} N^{2} \sin ^{2} \varphi_{0}
$$

where $N$ is the refractive index of the flint-glass, $\varphi_{0}$ is the incident angle.

The equation for the magnetic field component $\left|H_{y}\right|$ can be transformed into the following selfconjugated form :

$$
\begin{aligned}
& \frac{\mathrm{d}}{\mathrm{d} z}\left(g_{11} \frac{\mathrm{d}\left|H_{y}\right|}{\mathrm{d} z}\right)+ \\
& \quad+\frac{\omega^{2}}{c^{2}}\left[\frac{g_{11} n_{0}^{2} n_{\mathrm{e}}^{2}-N^{2} \sin ^{2} \varphi_{0}}{g_{11} n_{0}^{2} n_{\mathrm{e}}^{2}}\right]\left|H_{y}\right|=0
\end{aligned}
$$

$\left|H_{y}\right|$ is a monotonically increasing function for $g_{11}>N^{2} \sin ^{2} \varphi_{0}$ with a maximum in the middle of the liquid crystal cell for a symmetric case.

The authors however are interested in ATIR behaviour of the electromagnetic wave corresponding to exponential variations of the field components.
With the aid of the substitution :

$$
\left|H_{y t}\right|=\left|H_{y}\right| \exp \int_{0}^{z}\left[(1 / 2) \frac{\mathrm{d} g_{11}}{\mathrm{~d} z} / g_{11}\right] \mathrm{d} z
$$

the differential equation (4) is transformed to the following convenient normal form :

$$
\frac{\mathrm{d}^{2}\left|H_{y \mathrm{t}}\right|}{\mathrm{d} z^{2}}+p(z)\left|H_{y \mathrm{t}}\right|=0
$$

where

$$
p(z)=\frac{\omega^{2}}{c^{2}} f(z)+g(z)
$$

and

$$
\begin{aligned}
& f(z)=\frac{g_{11} n_{0}^{2} n_{\mathrm{e}}^{2}-N^{2} \sin ^{2} \varphi_{0}}{n_{0}^{2} n_{\mathrm{e}}^{2} g_{11}^{2}} \\
& g(z)=(1 / 4)\left[\frac{\mathrm{d} g_{11} / \mathrm{d} z}{g_{11}}\right]^{2}-(1 / 2) \frac{\mathrm{d}^{2} g_{11} / \mathrm{d} z^{2}}{g_{11}} .
\end{aligned}
$$

The propagation of a TM wave in one nonhomogeneous anisotropic medium presents a very complicated problem. It has been solved as pointed out with the aid of numerical methods. Unfortunately this in the authors opinion is not convenient for obtaining one possible relation between the angle of incidence $\varphi_{0}$ and the all parameters of the liquid crystal entering in the problem for ATIR.

In order to obtain the ATIR condition one standard method applied in a number of books and papers (see e.g. [41]) for one nonhomogeneous media is followed : the differential equation describing the variations of the magnetic component $H_{y}$ for one TM wave is transformed into a normal wave form and is solved in the approximation of geometrical optics. The plane where this solution is not valid gives usually one exponentially evanescent wave in the media depth under investigation and one standing wave in the opposite direction. From this case one can obtain the ATIR condition as well.

The magnetic component $\left|H_{y}\right|$ is considered in order to understand the importance of the value of the incident angle $\varphi_{0}$ :

$$
\left|H_{y}\right|=\left|H_{y t}\right| \exp (-1 / 2) \int_{0}^{z}\left(\frac{\mathrm{d} g_{11}}{\mathrm{~d} z} / g_{11}\right) \mathrm{d} z
$$

It is clear that $\left|H_{y}\right|$ depends not only on the variation of $\left|H_{y t}\right|$ along $Z$ but also on the sign of the integral in the exponent :

$$
\begin{aligned}
& (-1 / 2) \int_{0}^{z}\left(\mathrm{~d} g_{11} / \mathrm{d} z\right)\left(1 / g_{11}\right) \mathrm{d} z= \\
& \quad=\int_{0}^{z} \frac{\left(n_{\mathrm{e}}^{2}-n_{0}^{2}\right) \sin \theta \cos \theta}{n_{\mathrm{e}}^{2} \cos ^{2} \theta+n_{0}^{2} \sin ^{2} \theta} \cdot \frac{\mathrm{d} \theta}{\mathrm{d} z} \mathrm{~d} z
\end{aligned}
$$


For a symmetrically deformed homeotropic liquid crystal $\theta$ and $\mathrm{d} \theta / \mathrm{d} z$ have the same signs and in general the function multiplying $\left|H_{y t}\right|$ is increasing exponentially.

By varying the angle $\varphi_{0}$ (e.g. the value of the function $p(z)$ ) it is possible to obtain an exponential solution for the magnetic field component $\left|H_{y t}\right|$.

This problem may be solved for one arbitrary function $p(z)$ only for the important case where the propagation conditions for a component $\left|H_{y t}\right|$ approximate those of geometrical optics (Landau and Lifshitz [42]), Brehovskikh [43]. Due to the term (9) this solution is intermediate between the cases for the wave optics and for the geometrical optics. In what follows it shall be assumed that the function $p(z)$ is real. In equation (7) the quantity $2 \pi(p(z))^{1 / 2}$ plays the part of wavelength in the $Z$-direction. The approximation of geometrical optics for the component $\left|H_{y t}\right|$ corresponds to the inequality :

$$
\frac{\mathrm{d}}{\mathrm{d} z} \frac{1}{(p(z))^{1 / 2}}<1
$$

and the two independent solutions of equation (7) are of the form :

$$
\frac{\text { constant }}{|p(z)|^{1 / 4}} \exp \pm \int|p(z)|^{1 / 2} \mathrm{~d} z
$$

for the case $p(z)<0$.
It is clear that the phase variation of the components $H_{y}, E_{x}$ and $E_{z}$ along $Z$ are determined from the approximate solutions of equation (7). The amplitudes of $H_{y}, E_{x}$ and $E_{z}$ according to (6) and (10) are with an exponential variation along $Z$.

The condition (10) is certainly not fulfilled near any point where $p(z)=0$. This case in investigated in a number of papers and books (see for example [42]). Around the plane where $p(z)=0$ the component $\left|H_{y \mathrm{t}}\right|$ is a stationary $\left(z \lesssim z_{\mathrm{m}}\right)$ or an exponentially damped component penetrating into the region $z \gtrsim z_{\mathrm{m}}$ (see the Fig. 2).

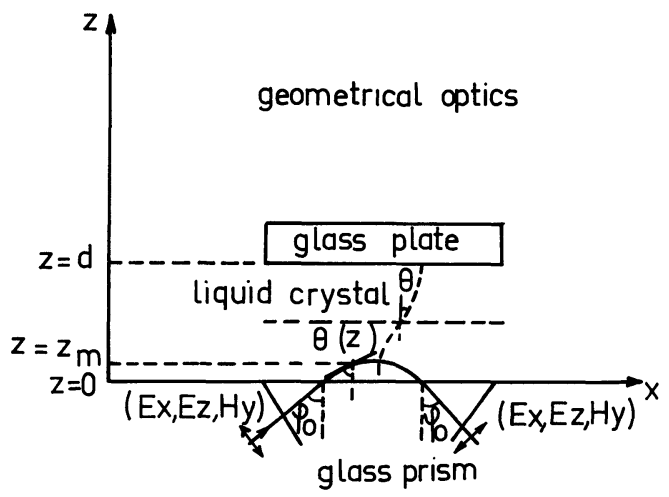

Fig. 2. - ATIR from the plane $z=z_{\mathrm{m}}$ in a deformed initially homeotropic nematic liquid crystal : geometrical optics.

The condition $p(z)=0$ gives the important connection between the parameter $N^{2} \sin ^{2} \varphi_{0}$, the deformation in the liquid crystal and all constants in the problem investigated :

$$
\frac{g_{11} k^{2} n_{0}^{2} n_{\mathrm{e}}^{2}-\beta^{2}}{\cdot n_{0}^{2} n_{\mathrm{e}}^{2} g_{11}^{2}}-(1 / 4)\left[\frac{\mathrm{d} g_{11} / \mathrm{d} z}{g_{11}}\right]^{2}-(1 / 2) \frac{\mathrm{d}}{\mathrm{d} z} \frac{\mathrm{d} g_{11} / \mathrm{d} z}{g_{11}}=0
$$

where $k^{2}=\omega^{2} / c^{2}, \beta^{2}=\left(\omega^{2} / c^{2}\right) N^{2} \sin ^{2} \varphi_{0}$.

Using the tensor component $g_{11}$ from (3) one can obtain the following relation :

$$
\begin{aligned}
N^{2} \sin ^{2} \varphi_{0}= & \left(n_{\mathrm{e}}^{2} \cos ^{2} \theta+n_{0}^{2} \sin ^{2} \theta\right) \times \\
& \times\left\{1+\frac{n_{\mathrm{e}}^{2}-n_{0}^{2}}{n_{0}^{2} n_{\mathrm{e}}^{2} k^{2}}\left[\frac{\cos ^{2} \theta \sin ^{2} \theta\left(n_{\mathrm{e}}^{2}-n_{0}^{2}\right)}{\left(n_{\mathrm{e}}^{2} \cos ^{2} \theta+n_{0}^{2} \sin ^{2} \theta\right)}\left(\frac{\mathrm{d} \theta}{\mathrm{d} z}\right)^{2}+\cos 2 \theta\left(\frac{\mathrm{d} \theta}{\mathrm{d} z}\right)^{2}+\frac{1}{2} \sin 2 \theta \frac{\mathrm{d}^{2} \theta}{\mathrm{d} z^{2}}\right]\right\} .
\end{aligned}
$$

This condition is valid for every plane between the boundary $z=0$ and the middle of the liquid crystal cell.

The following result :

$$
\begin{aligned}
N^{2} \sin ^{2} \varphi_{0}=\left(n_{\mathrm{e}}^{2} \cos ^{2} \theta+n_{0}^{2} \sin ^{2} \theta\right)\{1 & +(1 / 16)\left(\lambda_{0} / \lambda d\right)^{2} \frac{\sin ^{2} 2 \theta\left(n_{\mathrm{e}}^{2}-n_{0}^{2}\right)}{n_{0}^{2} n_{\mathrm{e}}^{2}} \times \\
\times & {\left.\left[\frac{\sin ^{2} 2 \theta\left(n_{\mathrm{e}}^{2}-n_{0}^{2}\right)}{4\left(n_{\mathrm{e}}^{2} \cos ^{2} \theta+n_{0}^{2} \sin ^{2} \theta\right)}+\cos 2 \theta-h^{2} \lambda^{2}\right]\right\}, \quad z=0 }
\end{aligned}
$$

is obtained from (13) using the notations of Nehring et al. [23] at the boundary $z=0$ (Fig. 1):

$$
\begin{gathered}
\frac{\mathrm{d} \theta}{\mathrm{d} z}=\left(\frac{\pi}{\hat{\lambda} d}\right) \sin \theta \cos \theta=(1 / b) \sin \theta \cos \theta, \quad \frac{\mathrm{d}^{2} \theta}{\mathrm{d} z^{2}}=\frac{\pi^{2} h^{2}}{d^{2}} \sin \theta \cos \theta \\
b=\left(K / W_{\mathrm{s}}\right), \quad \pi^{2} h^{2}=\left(\frac{|\Delta \varepsilon|}{4 \pi K}\right) E^{2}, \quad h=\left(U / U_{0}\right), \quad U_{0}=\left(\frac{4 \pi K}{|\Delta \varepsilon|}\right)^{1 / 2}
\end{gathered}
$$


where $W_{\mathrm{s}}$ is the surface energy constant, $K$, the mean elastic constant, $\Delta \varepsilon$, the low frequency dielectric anisotropy, $E$, the applied electric field, $U_{0}$, the voltage threshold corresponding to strong anchoring of the liquid crystal molecules with the surfaces when $W_{\mathrm{s}} \rightarrow \infty, d$, the thickness of the liquid crystal layer, $b$, the de Gennes coherent strength length.

One evaluation of (14) shows that $p(z)<1$ when :

$$
(1 / 16)\left(\lambda_{0} / \lambda d\right)^{2}<1
$$

e.g. for thicker cells, weak anchoring of the liquid crystal molecules and liquid crystals possessing weak optical anisotropy.

Conditions of such type for one nonhomogeneously isotropic media are usually a sufficient requirement for ohtaining ATIR. The answer however for a deformed nematic liquid crystal which presents one nonhomogeneous and anisotropic media is more difficult.

Let us now investigate more carefully the behaviour of the TM wave under investigation and the reflectivity properties. The variations of the magnetic field component can be obtained in the following manner :

a) In the region of the plane $z=z_{\mathrm{m}}$ (Fig. 2) where $p(z)=0$ one must investigate the equation (see e.g. Jeffreys and Jeffreys [44], Brekhovskikh [43], Olver [45]) :

$$
\frac{\mathrm{d}^{2}\left|H_{y \mathrm{t}}\right|}{\mathrm{d} z^{2}}-(\mathrm{d} p(z) / \mathrm{d} z)\left(z_{\mathrm{m}}\right)\left(z-z_{\mathrm{m}}\right)\left|H_{\mathrm{yt}}\right|=0
$$

with solutions $A_{i}(\widetilde{z})$ and $B_{i}(\widetilde{z})$ for $z>z_{\mathrm{m}}$ and $A_{i}(i \widetilde{z})$ and $B_{i}(i \widetilde{z})$ for $z<z_{\mathrm{m}}$ where :

$$
\tilde{z}=-(\mathrm{d} p(z) / \mathrm{d} z)\left(z_{\mathrm{m}}\right)\left(z-z_{\mathrm{m}}\right) .
$$

The Airy functions are reasonable approximations for solutions of the equation (7) only near the plane $z=z_{\mathrm{m}}$. The solutions for $z \neq z_{\mathrm{m}}$ can be represented with the following integral equations which can be solved by the method of successive iterations (see e.g. Smirnov [46]) :

a) $0 \leqslant z \leqslant z_{\mathrm{m}}-\varepsilon, \quad \varepsilon \ll 1$

$$
\begin{gathered}
v=u_{0}(z)^{1 / 2}\left|H_{y \mathrm{t}}\right| \\
v(\tau)=C_{1} \sin \frac{\omega}{c} \tau+C_{2} \cos \frac{\omega}{c} \tau+\frac{c}{\omega} \int_{0}^{\tau} \sin \left[\frac{\omega}{c}(\tau-t)\right] s(t) v(t) \mathrm{d} t
\end{gathered}
$$

where

$$
\begin{aligned}
f(z) & =u_{0}^{2}(z)=\frac{g_{11} n_{0}^{2} n_{\mathrm{e}}^{2}-N^{2} \sin ^{2} \varphi_{0}}{g_{11}^{2} n_{0}^{2} n_{\mathrm{e}}^{2}}, \quad N^{2} \sin ^{2} \varphi_{0}<g_{11} n_{0}^{2} n_{\mathrm{e}}^{2} \\
g(z) & =(1 / 4)\left(\mathrm{d} g_{11} / \mathrm{d} z\right)^{2}\left(1 / g_{11}^{2}\right)-(1 / 2)\left(\mathrm{d}^{2} g_{11} / \mathrm{d} z^{2}\right)\left(1 / g_{11}\right), \\
s(\tau) & =(1 / 4)\left(\mathrm{d}^{2} f / \mathrm{d} z^{2}\right)\left(1 / f^{2}\right)-(5 / 16)(\mathrm{d} f / \mathrm{d} z)^{2}\left(1 / f^{3}\right)-g(z) / f(z) \\
\tau & =\int_{0}^{z} u_{0}(t) \mathrm{d} t
\end{aligned}
$$

b) $z_{\mathrm{m}}+\varepsilon \leqslant z<d, \quad \varepsilon \ll 1$

$$
\begin{gathered}
v=\left(-u_{0}(z)\right)^{1 / 2}\left|H_{y t}\right| \\
v(\tau)=C_{1} \exp \left(\frac{\omega}{c} \tau\right)+C_{2} \exp \left(-\frac{\omega}{c} \tau\right)+\frac{c}{\omega} \int_{z_{\mathrm{m}}+\varepsilon}^{z} \sinh \left[\frac{\omega}{c}(\tau-t)\right] s(t) v(t) \mathrm{d} t
\end{gathered}
$$

where

$$
\begin{gathered}
f(z)=-u_{0}(z)^{2}=-\frac{g_{11} n_{0}^{2} n_{\mathrm{e}}^{2}-N^{2} \sin ^{2} \varphi_{0}}{g_{11}^{2} n_{0}^{2} n_{\mathrm{e}}^{2}} \\
N^{2} \sin ^{2} \varphi_{0}>g_{11} n_{0}^{2} n_{\mathrm{e}}^{2} \\
g(z)=(1 / 4)\left(\mathrm{d} g_{11} / \mathrm{d} z\right)^{2}\left(1 / g_{11}^{2}\right)-(1 / 2)\left(\mathrm{d}^{2} g_{11} / \mathrm{d} z^{2}\right)\left(1 / g_{11}\right), \\
s(\tau)=(1 / 4)\left(\mathrm{d}^{2} f / \mathrm{d} z^{2}\right)\left(1 / f^{2}\right)-(5 / 16)(\mathrm{d} f / \mathrm{d} z)^{2}\left(1 / f^{3}\right)+g(z) / f(z) \\
\tau=\int_{z_{\mathrm{m}}+\varepsilon}^{z}\left(-u_{0}(t)\right) \mathrm{d} t .
\end{gathered}
$$


Let us note that the integrals $\int_{0}^{z} u_{0}(t) \mathrm{d} t$ and $\int_{z_{\mathrm{m}}+\varepsilon}^{z}\left(-u_{0}(t)\right) \mathrm{d} t$ in the solutions (18) and (19) respectively are parameters.

The electric field components of the TM wave under investigation can be obtained from the Maxwell equations (1) :

$$
\begin{aligned}
& E_{x}=i \frac{c}{\omega}\left[\left(\mathrm{d} H_{y} / \mathrm{d} z\right) g_{11}+\frac{\omega}{c} i N \sin \varphi_{0} g_{13} H_{y}\right] \\
& E_{z}=i \frac{c}{\omega}\left[\left(\mathrm{d} H_{y} / \mathrm{d} z\right) g_{13}+\frac{\omega}{c} i N \sin \varphi_{0} g_{33} H_{y}\right]
\end{aligned}
$$

and the solution for the magnetic field component $H_{y}$ :

$$
\begin{array}{r}
H_{y}=\left|H_{y \mathrm{t}}\right| \exp \left[-\int_{0}^{z}(1 / 2)\left(\mathrm{d} g_{11} / \mathrm{d} z\right)\left(1 / g_{11}\right) \mathrm{d} z\right] \exp \left[-i \frac{\omega}{c} N \sin \varphi_{0} \int_{0}^{z}\left(g_{13} / g_{11}\right) \mathrm{d} z\right] \times \\
\times \exp \left[\left(-i \frac{\omega}{c} N \sin \varphi_{0} x\right)\right] .
\end{array}
$$

The investigations of the reflectivity properties of the liquid crystal layer from these different solutions in the different regions of the liquid crystal layer is a very difficult problem. The WBK approximation however gives a key to the solution when the value of the important parameter $N \sin \varphi_{0}$ determines $p\left(z_{\mathrm{m}}\right)<0$ :

$$
\frac{\mathrm{d}^{2}\left|H_{y t}\right|}{\mathrm{d} z^{2}}-\left[\frac{\omega^{2}}{c^{2}} f(z)+g(z)\right]\left|H_{y t}\right|=0
$$

where

$$
\begin{aligned}
& f(z)=\frac{N^{2} \sin ^{2} \varphi_{0}-g_{11} n_{0}^{2} n_{\mathrm{e}}^{2}}{n_{0}^{2} n_{\mathrm{e}}^{2} g_{11}^{2}} \\
& g(z)=-(1 / 4)\left(\mathrm{d} g_{11} / \mathrm{d} z\right)^{2}\left(1 / g_{11}\right)^{2}+(1 / 2)\left(\mathrm{d}^{2} g_{11} / \mathrm{d} z^{2}\right)\left(1 / g_{11}\right) .
\end{aligned}
$$

It is important to consider the surface region and the region in the middle of the liquid crystal layer.

3. Surface region of the liquid crystal layer. - The WBK method yields the following solutions for the equation (22) [47] :

$$
H_{y t}=\exp \left[\frac{\omega}{c} \int u(z) \mathrm{d} z\right]
$$

where

$$
\begin{gathered}
u(z)=\sum_{v=0}^{\infty} u_{v}(z)\left(\frac{\omega}{c}\right)^{-v} \\
u_{0}= \pm f(z)^{1 / 2}, u_{1}=-\left(\mathrm{d} u_{0} / \mathrm{d} z\right)\left(1 / 2 u_{0}\right), \\
u_{2}=\left[g(z)-\left(\mathrm{d} u_{1} / \mathrm{d} z\right)-u_{1}^{2}\right]\left(1 / 2 u_{0}\right) \\
u_{v+1}=\left(-1 / 2 u_{0}\right) \cdot\left(\mathrm{d} u_{v} / \mathrm{d} z+\sum_{p=1}^{v} u_{p} u_{v+1-p}\right), \quad v \geqslant 2 .
\end{gathered}
$$

It is easy to calculate the TM components $E_{x}, E_{z}$ and $H_{y}$ in the liquid crystal layer (a medium II) with the aid of the solution for the transformed magnetic field component $\left|H_{y t}\right|$ from (20) and (21).

$$
\begin{aligned}
& H_{y}^{\mathrm{II}}=\left|H_{y \mathrm{t}}\right| R \\
& E_{x}^{\mathrm{II}}=i \frac{c}{\omega}\left[(-1 / 2)\left(\mathrm{d} g_{11} / \mathrm{d} z\right)\left|H_{y \mathrm{t}}\right|+g_{11}\left(\mathrm{~d}\left|H_{y \mathrm{t}}\right| / \mathrm{d} z\right)\right] R \\
& E_{z}^{\mathrm{II}}=i \frac{c}{\omega}\left[(-1 / 2)\left(\mathrm{d} g_{11} / \mathrm{d} z\right)\left(g_{13} / g_{11}\right)\left|H_{y \mathrm{t}}\right|+\frac{\omega}{c} \frac{i N \sin \varphi_{0}}{g_{11} n_{0}^{2} n_{\mathrm{e}}^{2}}\left|H_{y \mathrm{t}}\right|+g_{13}\left(\mathrm{~d}\left|H_{y \mathrm{t}}\right| / \mathrm{d} z\right)\right] R
\end{aligned}
$$


where

$$
R=\exp \left[(-1 / 2) \int_{0}^{z}\left(\mathrm{~d} g_{11} / \mathrm{d} z\right)\left(1 / g_{11}\right) \mathrm{d} z-i \frac{\omega}{c} N \sin \varphi_{0} \int_{0}^{z}\left(g_{13} / g_{11}\right) \mathrm{d} z-i \frac{\omega}{c} N \sin \varphi_{0} x\right] .
$$

The values of the TM-wave components in the first medium - the flint-glass $60^{\circ}$ prism (I) and in the third medium - the glass plate (III) (Fig. 1) must be known for unit incident amplitude and a reflectivity coefficient $V_{\mathrm{TM}}$ :

$$
\begin{aligned}
& H_{y}^{\mathrm{I}}=\exp -i \frac{\omega}{c} N \sin \varphi_{0} x\left[\exp i \frac{\omega}{c} N \cos \varphi_{0} z+V_{\mathrm{TM}} \exp -i \frac{\omega}{c} N \cos \varphi_{0} z\right] \\
& E_{x}^{\mathrm{I}}=-\left(\cos \varphi_{0} / N\right) \exp -i \frac{\omega}{c} N \sin \varphi_{0} x\left[\exp i \frac{\omega}{c} N \cos \varphi_{0} z-V_{\mathrm{TM}} \exp -i \frac{\omega}{c} N \cos \varphi_{0} z\right] \\
& E_{z}^{\mathrm{I}}=-\left(\sin \varphi_{0} / N\right) \exp -i \frac{\omega}{c} N \sin \varphi_{0} x\left[\exp i \frac{\omega}{c} N \cos \varphi_{0} z+V_{\mathrm{TM}} \exp -i \frac{\omega}{c} N \cos \varphi_{0} z\right] .
\end{aligned}
$$

The electromagnetic TM-wave components in the third medium - the plate glass are given by the following expressions :

$$
\begin{aligned}
& H_{y}^{\mathrm{III}}=W_{\mathrm{TM}} \exp -i \frac{\omega}{c} N \sin \varphi_{0} x \exp i \frac{\omega}{c} n_{\mathrm{s}} \cos \varphi_{1} z \\
& E_{x}^{\mathrm{III}}=i W_{\mathrm{TM}}\left(\cos \varphi_{1} / n_{\mathrm{s}}\right) \exp -i \frac{\omega}{c} N \sin \varphi_{0} x \exp i \frac{\omega}{c} n_{\mathrm{s}} \cos \varphi_{1} z \\
& E_{z}^{\mathrm{III}}=-i W_{\mathrm{TM}}\left(N \sin \varphi_{0} / n_{\mathrm{s}}^{2}\right) \exp -i \frac{\omega}{c} N \sin \varphi_{0} x \exp i \frac{\omega}{c} n_{\mathrm{s}} \cos \varphi_{1} z
\end{aligned}
$$

where $W_{\mathrm{TM}}$ is the transmissivity coefficient and $\sin \varphi_{1}=\frac{\left(N \sin \varphi_{0}\right)}{n_{\mathrm{s}}}<1$ and

$$
\begin{aligned}
& H_{y}^{\mathrm{III}}=W_{\mathrm{TM}} \exp -i \frac{\omega}{c} N \sin \varphi_{0} x \exp -\frac{\omega}{c} \frac{\left(N^{2} \sin ^{2} \varphi_{0}-n_{\mathrm{s}}^{2}\right)^{1 / 2}}{n_{\mathrm{s}}} z \\
& E_{x}^{\mathrm{III}}=-\left(i / n_{\mathrm{s}}^{3}\right) W_{\mathrm{TM}}\left(N^{2} \sin ^{2} \varphi_{0}-n_{\mathrm{s}}^{2}\right)^{1 / 2} \exp -i \frac{\omega}{c} N \sin \varphi_{0} x \exp -\frac{\omega}{c} \frac{\left(N^{2} \sin ^{2} \varphi_{0}-n_{\mathrm{s}}^{2}\right)^{1 / 2}}{n_{\mathrm{s}}} z \\
& E_{z}^{\mathrm{III}}=\left(i / n_{\mathrm{s}}^{2}\right) N \sin \varphi_{0} W_{\mathrm{TM}} \exp -i \frac{\omega}{c} N \sin \varphi_{0} x \exp -\frac{\omega}{c} \frac{\left(N^{2} \sin ^{2} \varphi_{0}-n_{\mathrm{s}}^{2}\right)^{1 / 2}}{n_{\mathrm{s}}} z
\end{aligned}
$$

when the refractive index of the plate glass $n_{\mathrm{s}}$ is smaller than the parameter $N \sin \varphi_{0}$.

The real integral constants $C_{1}$ and $C_{2}$ and the complex reflectivity and transmissivity coefficients $V_{\mathrm{TM}}$ and $W_{\mathrm{TM}}$ may be obtained from the requirement for continuity of the components $H_{y}$ and $E_{x}$ :

$$
\begin{array}{cc}
H_{y}^{\mathrm{I}}=H_{y}^{\mathrm{II}} & H_{y}^{\mathrm{II}}=H_{y}^{\mathrm{III}} \\
E_{x}^{\mathrm{I}}=E_{x}^{\mathrm{II}} & E_{x}^{\mathrm{II}}=E_{x}^{\mathrm{III}} \\
z=0 & z=d
\end{array}
$$

For our purpose only the calculations of the real integral constants $C_{1}$ and $C_{2}$ and the modulus of the real coefficient $V_{\text {TM }}$ are sufficient.

The straightforward calculation leads to the following value of

$$
\left|V_{\mathrm{TM}}\right|=1-\left|(c / \omega) \frac{(-1 / 2) \mathrm{d} g_{11} / \mathrm{d} z\left|H_{y \mathrm{t}}\right|+g_{11}\left(\mathrm{~d}\left|H_{y \mathrm{t}}\right| / \mathrm{d} z\right)}{\left(\cos \varphi_{0} / N\right)}\right|, \quad z=0
$$

It is interesting to note that ATIR $\left(\left|V_{\mathrm{TM}}\right| \rightarrow 1\right.$ or $\left.\left|E_{x}\right|_{z=0} \rightarrow 0\right)$ is realized for the following variation of the magnetic field component $\left|H_{y t}\right|$ :

$$
\left|H_{y \mathrm{t}}\right|=\exp \int(1 / 2)\left(\mathrm{d} g_{11} / \mathrm{d} z\right)\left(1 / g_{11}\right) \mathrm{d} z
$$


This condition corresponds to $\left|H_{y}\right|=$ const. In our case $\left|H_{y}\right|$ is a complicated function of $z$ and ATIR condition will be obtained for the case of : WBK approximation :

$$
(-1 / 2)\left(\mathrm{d} g_{11} / \mathrm{d} z\right)-(1 / 2)\left(g_{11} / u_{0}\right)\left(\mathrm{d} u_{0} / \mathrm{d} z\right)-\frac{\omega}{c} g_{11}\left(u_{0}+\left(\frac{c}{\omega}\right)^{2} u_{2}+\cdots\right) \cdot \frac{C_{2}-C_{1}}{C_{2}+C_{1}}=0, \quad z=0 .
$$

The removal of the glass plate (in our case the lower plate) leads to the elimination of the integral constant $C_{1}: C_{1}=0$. This means that we regard one infinite liquid crystal medium. The ATIR condition for this particular case can be written in the following form :

$$
(-1 / 2)\left(\mathrm{d} g_{11} / \mathrm{d} z\right)-\left(g_{11} / 2\right)\left(1 / u_{0}\right)\left(\mathrm{d} u_{0} / \mathrm{d} z\right)-\frac{\omega}{c} g_{11}\left(u_{0}+\left(\frac{c}{\omega}\right)^{2} u_{2}+\cdots\right)=0 . \quad z=0,
$$

This condition should be valid for finite liquid crystal as well when the ratio $C_{1} / C_{2}$ is smaller than unity :

$$
\left(C_{1} / C_{2}\right)<1 .
$$

This ratio has the simplest form for one symmetrically deformed nematic layers :

$$
\left(C_{1} / C_{2}\right)=-\frac{\exp \int_{0}^{d}-2 \frac{\omega}{c}\left(u_{0}+\left(\frac{c}{\omega}\right)^{2} u_{2}+\cdots\right) \mathrm{d} z}{1+(c / \omega)\left(n_{\mathrm{s}} / \cos \varphi_{1}\right)\left(\mathrm{d} g_{11} / \mathrm{d} z\right)}, \quad z=d
$$

when $\sin \varphi_{1}=\left(N \sin \varphi_{0} / n_{\mathrm{s}}\right)<1$ and

$$
\left(C_{1} / C_{2}\right)=-\frac{\exp \int_{0}^{d}-2 \frac{\omega}{c}\left(u_{0}+\left(\frac{c}{\omega}\right)^{2} u_{2}+\cdots\right) \mathrm{d} z}{1+(c / \omega)\left[\left(N^{2} \sin ^{2} \varphi_{0}-n_{\mathrm{s}}^{2}\right)^{-1 / 2}\right] n_{\mathrm{s}}^{3}\left(\mathrm{~d} g_{11} / \mathrm{d} z\right)}, \quad z=d,
$$

when $\sin \varphi_{1}=\left(N \sin \varphi_{0} / n_{\mathrm{s}}\right)>1$.

One rough estimate for the value of the integral in the exponential function gives (see the results obtained by Rivière [17]) :

$$
\exp \int_{0}^{d}-2 \frac{\omega}{c}\left(u_{0}+\left(\frac{c}{\omega}\right)^{2} u_{2}+\cdots\right) \mathrm{d} z<\exp \left(-2 d / d_{\mathrm{p}}\right)
$$

where the penetration depth of the evanescent wave $d_{\mathrm{p}}$ was calculated by Rivière to be around $4 \mu k$. The real penetration depth of the evanescent wave is however in the range of the length of the incident wave [48] for the variation of the effective refractive index of the liquid crystal (see e.g. the results of $\mathrm{Hu}$ et al. [32]) : ATIR condition for the deformation at the interface flint-glass-liquid crystal $(Z=0)$ is valid even more for the deformations inside the liquid crystal layer.

The ATIR condition (32) can be used when the following inequalities are valid :

$$
\begin{aligned}
& d \gg \lambda_{0} \\
& \left(2 \pi / \lambda_{0}\right)\left(n_{\mathrm{s}} / \cos \varphi_{1}\right)\left(\mathrm{d} g_{11} / \mathrm{d} z\right)<1, \quad z=d \\
& \left(2 \pi / \lambda_{0}\right)\left[\left(N^{2} \sin ^{2} \varphi_{0}-n_{\mathrm{s}}^{2}\right)^{-1 / 2}\right] n_{\mathrm{s}}^{3}\left(\mathrm{~d} g_{11} / \mathrm{d} z\right)<1, \quad z=d
\end{aligned}
$$

where $d$ is the thickness of the liquid crystal cell, $\lambda_{0}$, the length of the incident wave, $n_{\mathrm{s}}$, the refractive index of the plate-glass, $N$, the refractive index of the flint-glass prism, $\varphi_{0}$, the angle of incidence, $\varphi_{1}$, the angle of transmission from the lower plate of the liquid crystal cell.

The exact ATIR condition can be calculated numerically from the relation (31).

The validity for the ATIR wave optics condition :

$$
u_{0}=0
$$

increases for weak anchoring of the liquid crystal molecules with the surfaces $\left(\mathrm{d} g_{11} / \mathrm{d} z\right) \rightarrow 0$, for small deformations and for thicker liquid crystal cells $\left(C_{1} / C_{2}\right) \rightarrow 0$ as well.

The first approximation to ATIR wave optics condition from (32) :

$$
N^{2} \sin ^{2} \varphi_{0}=g_{11} n_{0}^{2} n_{\mathrm{e}}^{2}\left[1+\left((1 / 4)\left(\lambda_{0} / 2 \pi\right)\left(1 / g_{11}^{2}\right)\left(\mathrm{d} g_{11} / \mathrm{d} z\right)\right)^{2 / 3}\right],
$$


is sufficient for many cases when the thickness of the liquid crystal layer under investigation is much larger than the length of the propagation TM wave (see also the inequalities (37)!).

The condition (39) with the notation of Nehring et al. (Rapini's type surface energy) [23] has the form :

$$
N^{2} \sin ^{2} \varphi_{0}=\left(n_{\mathrm{e}}^{2} \cos ^{2} \theta+n_{0}^{2} \sin ^{2} \theta\right)\left\{1+\left(\frac{\lambda_{0} n_{0}^{4} n_{\mathrm{e}}^{4}\left(n_{\mathrm{e}}^{2}-n_{0}^{2}\right) \sin ^{2} 2 \theta}{32 \lambda d\left(n_{\mathrm{e}}^{2} \cos ^{2} \theta+n_{0}^{2} \sin ^{2} \theta\right)^{2}}\right)^{2 / 3}\right\}, \quad z=0
$$

where $\left(\lambda_{0} / \lambda d\right)=\left(\lambda_{0} / b\right)(1 / \pi)$ and $b$ is de Gennes' extrapolation strength length.

One crude estimate of the relation (40) shows the possible application of the ATIR wave optics condition :

$$
N^{2} \sin ^{2} \varphi_{0} \simeq n_{\mathrm{e}}^{2} \cos ^{2} \theta+n_{0}^{2} \sin ^{2} \theta
$$

for $\lambda_{0} / b \sim 1$.

This approximate equality corresponds to one surface energy $W_{\mathrm{s}}$ in the range of $10^{-2} \mathrm{erg} / \mathrm{cm}^{2}$. One can anticipate that the ATIR wave optics condition is applicable no more for strong anchoring of the liquid crystal when :

$$
W_{\mathrm{s}}>5 \times 10^{-2} \mathrm{erg} / \mathrm{cm}^{2}
$$

In all cases one must follow the process of selfconsistence : e.g. the solution of the problem with ATIR wave optics condition and the verification of the results obtained from (32) and (37). For the case of strong deformations in the boundary regions (strong anchoring and high electric fields) the other terms in the relation (32) should be taken into account.

The comparison between the ATIR conditions (14) (see also Hinov [49]) and (40) shows that the condition for exponential variations of the TM-wave components expressed with the relation (14) is a weak requirement for the case of strong anchoring and thin liquid crystal cells.

The ATIR conditions (40) and (31) are more exact and lead to the total reflection of the light from the liquid crystal $:\left|V_{\mathrm{TM}}\right| \rightarrow 0$.

4. Middle region of the liquid crystal layer. Prevailing in this region are the second order derivatives of the deformation angle $\theta$ with respect the coordinate $Z$ which are usually weak. More applicable in this case is the ATIR wave optics condition (41).

It is interesting to note that the ATIR condition from the middle of the symmetric liquid crystal cell allows a very simple determination of the maximal deformation angle when the refractive index of the lower glass plate is sufficient large (see Eq. (28a)).

The comparison between the deformations in the surface and middle regions of the liquid crystal may give one qualitative estimate of the type of the surface energy (the groups working with weakly anchored liquid crystal layer now use Rapini's type surface energy [23]).
It is interesting to obtain the condition for ATIR from the view point of geometrical optics. This is easily done from the magnetic field dependence $H_{y}=\left|H_{y}\right| \exp i b z$ when the phase constant along $Z$ is zero :

$$
N^{2} \sin ^{2} \varphi_{0}=\frac{n_{0}^{2} n_{\mathrm{e}}^{2}}{n_{0}^{2} \cos ^{2} \theta+n_{\mathrm{e}}^{2} \sin ^{2} \theta}=n_{\mathrm{eff}}^{2}
$$

As was pointed out by Brehovskikh [43] the condition from geometrical optics are valid if the following inequality is realized :

$$
\frac{\lambda_{0}}{4 \cos ^{2} \theta} \frac{\mathrm{d}}{\mathrm{d} z}\left(\cos \theta / n_{\text {eff }}\right)<1 .
$$

The comparison between the conditions (41) and (43) for ATIR leads to the following relation at small angles :

$$
\frac{\sin \theta_{\text {wave optics }}}{\sin \theta_{\text {geometrical optics }}} \sim n_{\mathrm{e}} / n_{0}
$$

Equations (41) and (43) can be applied for every point in the bulk of the deformed liquid crystal if the condition $\lambda_{0} / b \lesssim 1$ is fulfilled (see (41)).

The relations (41) and (43) were solved with respect to the deformation angle $\theta$ :

$$
\begin{aligned}
& \theta=\arcsin \left[\frac{n_{\mathrm{e}}^{2}-N^{2} \sin ^{2} \varphi_{0}}{n_{\mathrm{e}}^{2}-n_{0}^{2}}\right]^{1 / 2} \\
& \theta=\arcsin \left[\frac{n_{0}^{2}}{n_{\mathrm{e}}^{2}-n_{0}^{2}}\right]^{1 / 2}\left[\frac{n_{\mathrm{e}}^{2}}{N^{2} \sin ^{2} \varphi_{0}}-1\right]^{1 / 2}
\end{aligned}
$$

These results have strong temperature dependence determined by variations of the anisotropic refractive indices.

The deformation angles were calculated for MBBA using Brunet-Germain [22] data for the refractive indices (Figs. 3 and 4). These results show the sharp temperature dependence especially when the deformations are small and the temperatures high. The largest difference between the two solutions (46) and (47) as seen from the curves are at $45^{\circ}$. The same result was obtained by Rivière et al. [18]. 


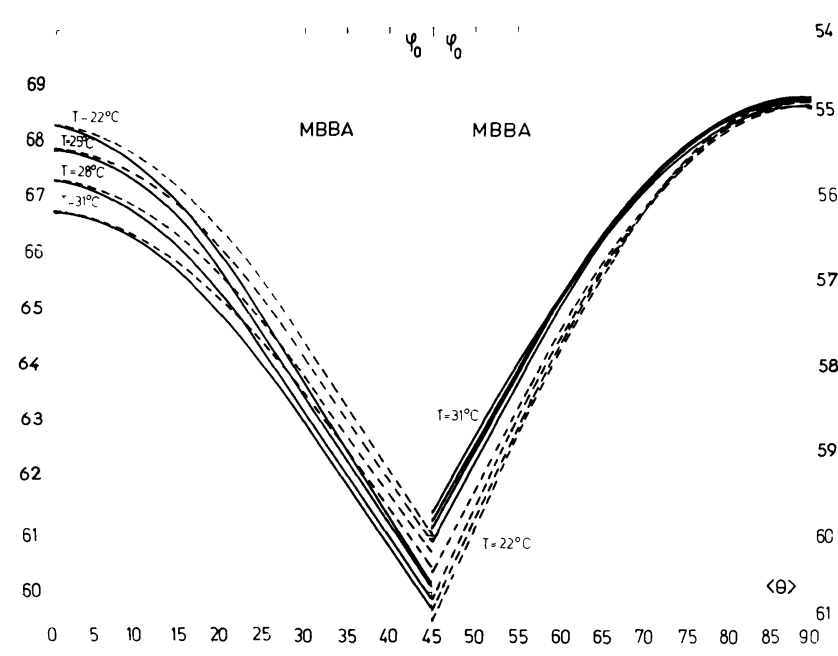

Fig. 3. - Deformation angles $\theta$ at ATIR calculated for MBBA from the Brunet-Germains [22] refractive indices data $\left(T=22-31{ }^{\circ} \mathrm{C}\right)$ versus the incident angle $\varphi_{0}$. Solid curves : geometrical optics calculations; dashed curves : wave optics calculations.

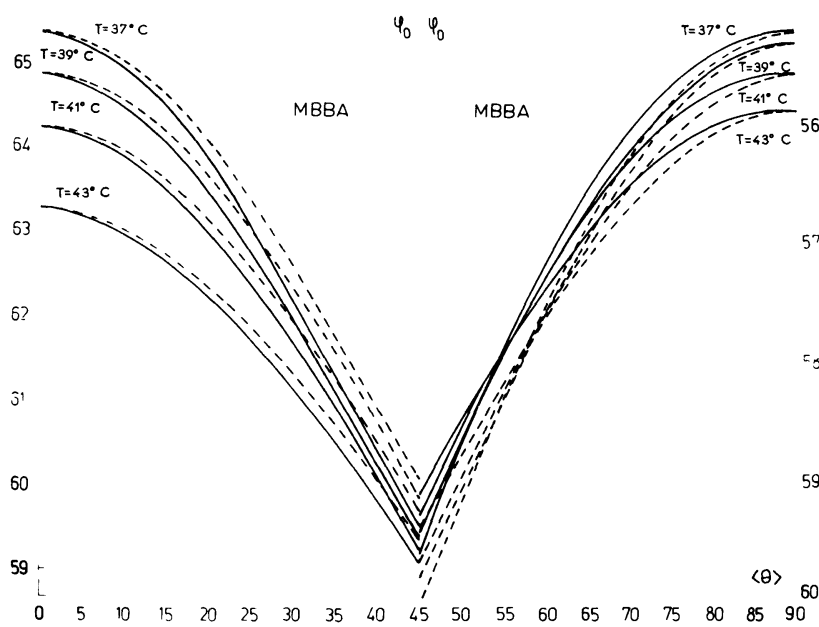

Fig. 4. - Deformation angles $\theta$ at ATIR calculated for MBBA from the Brunet-Germains [22] refractive indices data $\left(T=37-43^{\circ} \mathrm{C}\right)$ versus the incident angle $\varphi_{0}$. Solid curves : geometrical optics calculations ; dashed curves : wave optics calculations.

For clarification we drew the deformations in $5^{\circ}$ steps in the plane $\left(T, \varphi_{0}\right)$ (Figs. 5 and 6$)$. The sensitivity of the ATIR as pointed out in figures 3-6 is smaller for deformations in the range $0^{\circ}-15^{\circ} ; 75^{\circ}-90^{\circ}$ and larger for the remaining values.

The depth of penetration of the evanescent wave along $Z$ in the geometrical optics conditions has the known form :

$$
\begin{array}{r}
d_{\mathrm{p}}=\left(\lambda_{0} / 2 \pi\right)\left[\left[\frac{N \sin \varphi_{0}}{(1 / t) \int_{0}^{t} n_{\text {eff }} \mathrm{d} z}\right]^{2}-1\right]^{-1 / 2} \times \\
\times\left(\frac{1}{t}\right) \int_{0}^{t} n_{\text {eff }} \mathrm{d} t
\end{array}
$$

where $n_{0} \leqslant(1 / t) \int_{0}^{t} n_{\text {eff }} \mathrm{d} z \leqslant n_{\mathrm{e}}$ (see e.g. the results

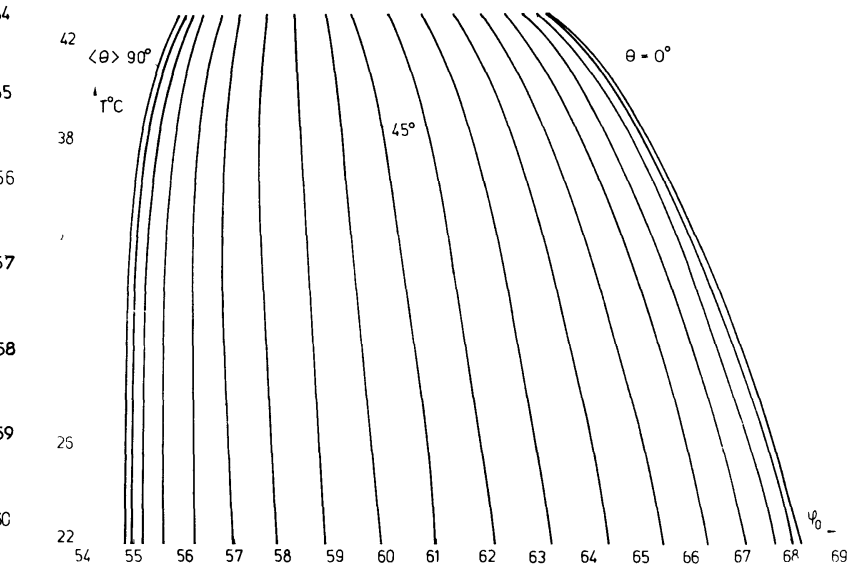

Fig. 5. - The deformation angles $\theta$ ( $5^{\circ}$ intervals) at ATIR for MBBA versus the incident angle $\varphi_{0}$ calculated from a wave optics point of view.

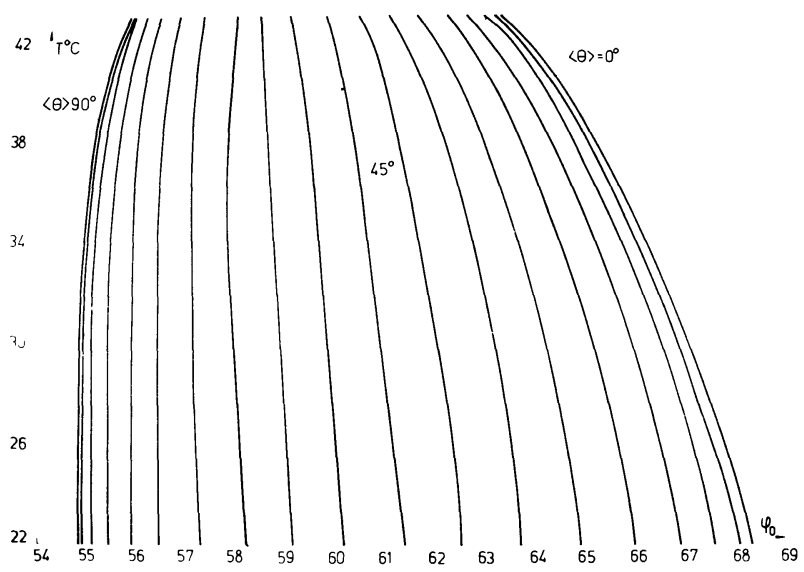

Fig. 6. - The deformation angles $\theta\left(5^{\circ}\right.$ intervals) at ATIR for MBBA versus the incident angle $\varphi_{0}$ calculated from a geometrical point of view.

obtained by $H$. Mada and Sh. Kobayashi [50]), $n_{0}$ is the ordinary refractive index, $\lambda_{0}$, the wave length of the incident wave in vacuum, $n_{\text {eff }}$, the index of refraction of liquid crystal along $Z$, which in deformed layers is a function of $Z, N$, the index of refraction of the first medium (usually flint-glass), $\varphi_{0}$, the angle of incidence of the TM wave. The penetration depth was obtained to be of the order of the length of the incident wave $: d_{\mathrm{p}} \sim \lambda_{0}$.

5. Experimental results [51]. - The measurements of the ATIR of surface deformation on the basis of the theoretical formulae obtained are presented in this part of the paper. In order to perform the experiment correct a number of requirements must be satisfied. Some of them are treated in the book by Harrick [52]. A choice must be made for the working angle or range of angles of incidence, the necessary materials, the surface preparation, etc... Other, very important requirements, are considered in the Thesis of D. Rivière [17]. 
Let us now list the more important requirements :

a) High quality of the internal reflection elements : adequate surface polish, good material, proper flatness, weak temperature dependence, etc.

b) High quality laser beam source.

c) Careful estimate of the optical properties of the material under investigation.

d) Careful estimate of experimental and measurement accuracy.

The experimental set-up is given on figure 7. One glass-flint $60^{\circ}$ prism very well polished (the roughness is in the range of $\lambda_{0} / 4$ ) with a high refractive index $N \sim 1.889$ and a common glass plate with a small

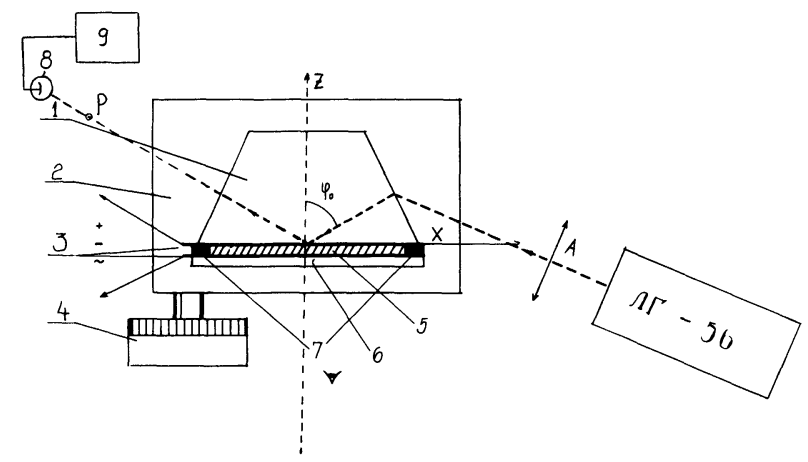

Fig. 7. - The experimental set-up for ATIR : 1. glass-flint $60^{\circ}$ prism, 2. rotational table, 3. d.c. or a.c. voltage generator, 4. a screw varying the angle of incidence, 5 . liquid crystal, 6. common glass plate, 7. teflon spacers, 8. photomultiplier, 9. $X-Y$ Brüel and Kjaer recorder working with a linear potentiometer $10-35 \mathrm{mV}$, type 2305

refractive index $\left(n_{\mathrm{s}} \sim 1.4\right)$ formed the liquid crystal cells with thicknesses between 12 and $45 \mu k$. The inner surfaces were coated with thin, semi-transparent chromium $200 \AA$ thick (the common glasses in some cases were coated with $\mathrm{SnO}_{2}$ as well). The investigated liquid crystal was MBBA at room temperature. The boundaries were rubbed and then treated with a thin lecithin layer giving nearly homeotropic orientation (when the layer is with a perfect homeotropic orientation the deformations start along $\theta$ and $\varphi$ and the problem is more complicated). Planar layers were achieved using rubbing technique. The liquid crystal deformations were obtained with d.c. or a.c. electric field excitation. A polarized $\mathrm{He}-\mathrm{Ne}\left(\lambda_{0}=632.8 \mathrm{~nm}\right)$ laser beam was incident on the flint-liquid crystal interface at an angle $\varphi_{0}$ :

$$
\varphi_{0}=60^{\circ} \pm \operatorname{arc} \sin (1 / N) \sin \tilde{\varphi}
$$

where $\tilde{\varphi}$ is the wave incident angle on the air-flint interface. For a range of angles $\varphi_{0}<\varphi<\pi / 2$, where $\varphi_{0}$ is the critical angle discussed in the theory, the beam is totally reflected at the flint-glass-liquid crystal interface.

The frequency band of the laser beam source LG-56 around the basic generated frequency corresponding to the length of $632.8 \mathrm{~nm}$ pointed out is $150 \mathrm{MHz}$. The divergence of the laser beam which is of the order of $10^{\prime}$ decreases two times after light entery in the flint-glass prism (see the book of Harrick [52]). Half of these $5 \mathrm{~min}$. are above the critical angle $\varphi_{0}$ at ATIR measurements. E.g. only $2.5^{\prime}$ are important for the accuracy of the ATIR experiment. Rivière [17] using a special optical construction reduced this divergence to the minimal value of $0.85^{\prime}$.

The theory presented in the first part of the paper is for a plane monochromatic wave. The utilized laser beam in effect can be approximated with the shape of a Gaussian beam (see papers [53]-[56]).

Still open for explanation is ATIR of a Gaussian beam from one inhomogeneous anisotropic medium such as the deformed nematic liquid crystal.

The discrepancy can be reduced however with regulation of the laser regime - the utilization of the central part of the laser beam which can be achieved at lower limit of the laser power.

The denser medium in our case is a flint-glass $60^{\circ}$ prism with a large refractive index $N \sim 1.889$.

The questions relating the influence of the electrodes and the additional deposed layers on the transmissivity properties of normal incident light on the twisted nematic layers were regarded e.g. by G. PaulMontgomery [57]-[59]. The thicknesses of the additional deposited layers : the semi-transparent chromium film and the lecithin in our case are much smaller than the wave length of the incident light : 200-500 $\AA$. This fact shows that these layers would disturb the ATIR conditions very little (see also the book of Harrick [52] or the paper [21]).

The influence of the semi-transparent electrode on the light reflectivity is significant when the refractive indices of the electrode and the liquid crystal are quite different [57]-[59]. Harrick [52] however pointed out that additional very thin layers with thicknesses much thinner than the penetration length of the evanescent wave are not significant for ATIR condition. This was confirmed in our experiment after cheking the ATIR condition of homogeneously oriented planar and homeotropic nematic MBBA layers with and without conductive films.

The rarer medium is a nematic liquid crystal with electrically controllable effective refractive index varying between the index of the ordinary wave $n_{0}$ and the index of the extraordinary wave $n_{\mathrm{e}}>n_{0}$. In the theory developed we neglected the strong temperature fluctuations of the nematic molecules around theirs equilibrium position (see the papers [60][66]). The scattering angle which is given in these works is several degrees around the forward direction of the laser beam. The great intensity of the thermal fluctuations is due to the little energy connected with the long-wave length orientational modes. The scattering angle between $10^{\prime}$ (the divergence of the laser beam) and $0.2^{\circ}$ (the lower limit of the thermal fluctuations) accordingly to the results of Langevin and 
Bouchiat [62] are created by static orientation defects in the sample. Another important feature is the depolarization of the light caused by $\varphi$, fluctuations of the nematic director. It is evident that the thermal fluctuations change the ATIR conditions. On the other hand, however, they clearly show the beginning of the total reflection of the light (see also [17] and [18]).

The theory was developed for one nonabsorbing media. This is correct for nematic liquid crystal illuminated with a laser beam in the wave length region 0.42 to $0.70 \mu k$ at room temperature [67]. The analysis given by Chang shows that the absorption in this region of the wave length is negligibly small $\left(k_{\|} \simeq k_{\perp} \sim 0.0005\right)$.

In authors opinion one accurate ATIR measurement must include :

a) Careful measurements of the transmitted or the reflected light intensity only of the central laser spot.

b) Careful estimate of the sensitivity of these measurements : e.g. the estimate of the ratio between the intensities of the light before and after the total reflection.

The reflected light intensity from the deformed nematic liquid crystal depends strongly on the type of polarization of the incident wave (TM or TE), the position of the nicols (when they are used), the part of the reflected light ray (central, middle, etc.), the interference between the forward and the backward waves (see [57]-[59]), the thermal fluctuations, the frequency and the rate of variation of the applied voltage deforming the liquid crystal, etc. For these reasons the authors decided to determine the beginning of ATIR when the laser spot transforms in a light trace at the flint-glass liquid crystal interface. Taking into consideration the sensitivities of the experimental measurements performed and the peculiarities of the experimental liquid crystal cell : the utilization of a lower glass-plate having a refractive index smaller than the liquid crystal refractive indices and consequently the concentration of the transmitted light mainly in the cell region.

The variations of the light spot intensities can be observed even with a naked eye and they are very sensitive to the variation of $\varphi>\varphi_{0}$ in the region of several minutes (see also papers [17], [18]).

We wish to point out the importance of the zero ATIR method based on the disappearance of the light transmissivity spot due to the thermal fluctuations of the liquid crystal molecules. The advantages of this method when compared with the measurements of the intensity of the reflected light as a function of the incident angle are obvious. The zero ATIR method gives the value of the critical angle with one measurement only with a sufficient accuracy at that in the range of $2-3^{\prime}$.

The values of the critical angles were obtained with the aid of this method (without measurements of the light intensities) for different liquid crystal orientations and voltages.
The mean values of the deformation angles in the region of the laser spot $\langle\theta\rangle$ based on the theoretical formulae (46) and (47) are shown on figures 9 and 8 for two MBBA cells at room temperatures with various orientation and d.c. or high frequency a.c. voltage excitation.

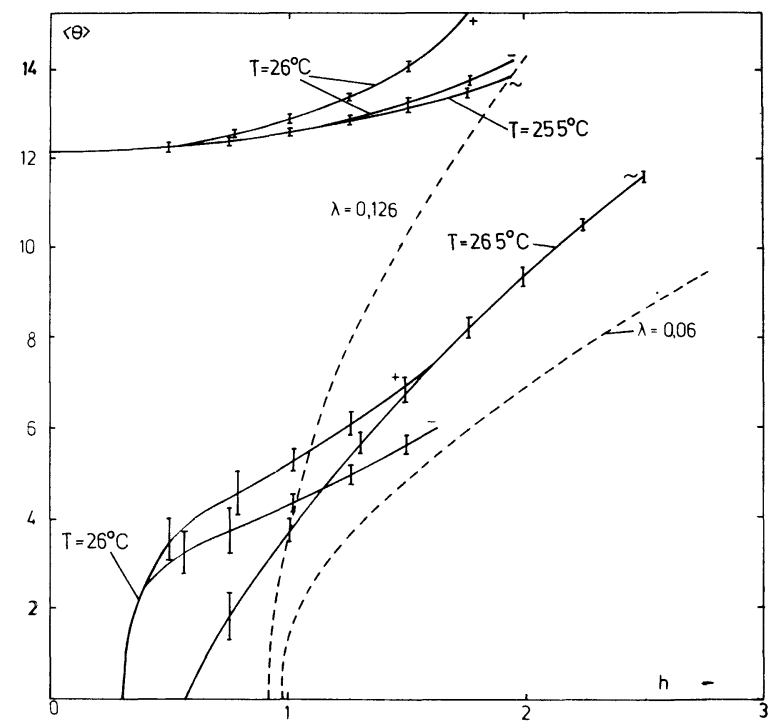

Fig. 8. - The mean deformation surface angle $\langle\theta\rangle$ versus the dimensionless parameter $h=U / U_{\text {th }}$ (d.c. or a.c. electric fields) for $12 \mu \mathrm{k}$ MBBA homeotropic (lower curves) and initially deformed (upper curves) layers. The flint-glass was coated with $\mathrm{Cr}$ and the common glass, with $\mathrm{SnO}_{2} ; T=21 \pm 0.1^{\circ} \mathrm{C}$. The dashed curves are theoretically calculated from the theory of Nehring et al. [23].

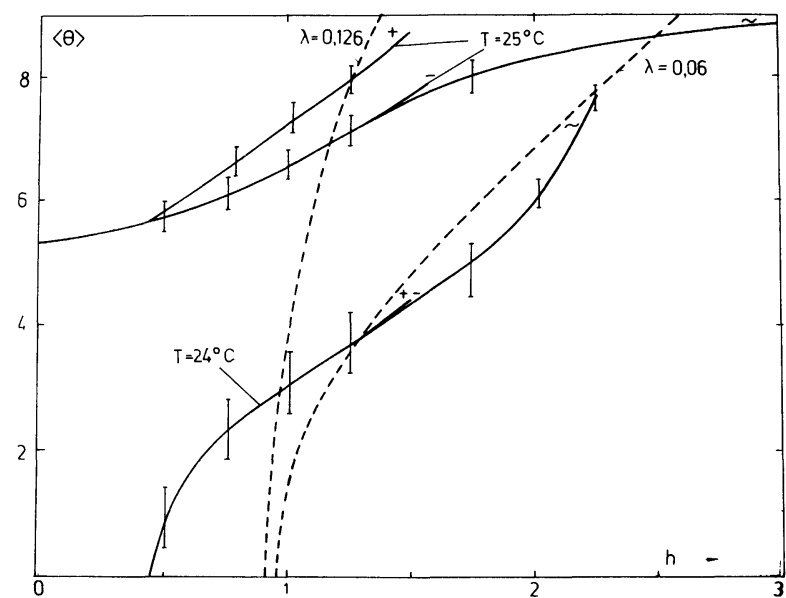

Fig. 9. - The mean deformation surface angle $\langle\theta\rangle$ versus the dimensionless parameter $h=U / U_{\text {th }}$ (d.c. or a.c. electric fields) - for $45 \mu \mathrm{k}$ MBBA homeotropic (lower curves) and initially deformed (upper curves) layers. The flint glass was coated with $\mathrm{Cr}$ and the common glass with $\mathrm{SnO}_{2} ; T=21 \pm 0.1^{\circ} \mathrm{C}$. The dashed curves are theoretically calculated from the theory of Nehring et al. [23].

ATIR measurements can also be disturbed by the nonhomogeneity of the liquid crystal orientation in the region of the laser light spot (see the thesis of Rivière [17]). Rivière pointed out that this fact leads to one uncertainity of $2^{\prime}$ for the measurements of the 
critical angle $\varphi_{0}$. This was confirmed in our experiment. The parasite action of the thermal fluctuations on the other hand can be neglected in the surface regions as pointed out by $\mathrm{Ch}$. $\mathrm{Hu}$ and Whinnery [64] and G. Labrunie and J. Robert [68].

We also performed measurements of the intensity of the reflected light at various incident angles : near ATIR condition and at $60^{\circ} \sim \varphi_{0}$ which corresponds to one deformation angle $\langle\theta\rangle$ in the range of $50^{\circ}$ : e.g. in this case the laser beam penetrates into the entire liquid crystal cell, at various positions of the nicols (or without an analyser), at various part of the reflected light ray (central, middle, etc.), etc... Standard experimental set-up with a photomultiplier and $X-Y$ Brüel and Kjaer recorder working with a linear potentiometer $10-35 \mathrm{mV}$ was used (Fig. 7). The experimental results obtained were very different depending on the conditions cited above. Some of them (Figs. 10-13) show in agreement with the results of T. Shimomura et al. [40] that this method is more sensitive when the angle between the liquid crystal molecules and the laser beam propagation is about $45^{\circ}$. The sensitivity of the zero ATIR condition showing the surface behaviour of the liquid crystal and the light reflectivity measurements showing the bulk liquid crystal orientation are of the same order only in this cited case. All other light reflectivity measurements are less sensitive as compared to the zero light-spot method.

The comparison between the figures 8 and 9 showing the variations of the surface orientation of the liquid crystal and figures 10-13 showing the bulk variations of the liquid crystal orientation in the authors' opinion show that the agreement is sufficient : e.g. the utilization of these two methods is complementary.

With the variation of $\varphi_{0}$ ATIR can be realized as was pointed out in every point inside the liquid crystal cell. Our reflectivity light measurements (Fig. 10) clearly show this fact : the oscillations are due to the interference between the forward and backward propagating waves [57]-[59]. The scale amplitude variations are determined by the penetration depth of the laser beam depending on the value of the applied a.c. electric field. In these cases the authors observed the deformations $0-45^{\circ}\left(\varphi_{0}=60^{\circ}\right)$ which propagate from the middle of the cell to the boundaries (the well known de Gennes coherence length $\zeta$ ) (see also [57][59]).

The accuracy of the calculated mean deformation angles $\langle\theta\rangle$ can be estimated from the accuracy of the temperature measurements $\left( \pm 0.1^{\circ} \mathrm{C}\right)$ and from the accuracy of the measurements of the ordinary and extraordinary indices of the liquid crystal

$$
\left(\Delta n_{\mathrm{e}}=\Delta n_{0}=\Delta n=5 \times 10^{-3}\right) .
$$

The experimental errors at these conditions are larger for deformation angles $0-15^{\circ}$ : around $1^{\circ}$ and smaller for all other values. They are smallest in the region of $45^{\circ}$ as pointed out by Rivière [17].

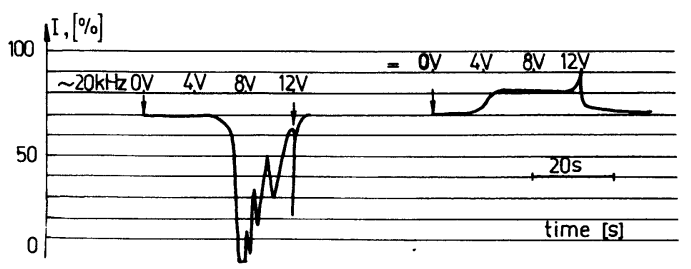

Fig. 10. - Light-reflectivity measurements at ATIR performed with a Brüel and Kjaer, type 2305, working with a linear potentiometer $10-35 \mathrm{mV}$ in $12 \mu \mathrm{k}$ MBBA layer. The flint-glass was coated with $\mathrm{Cr}$, the common glass, with $\mathrm{SnO}_{2}$. The left curve : ATIR in the liquid crystal depth : $\varphi_{0}=60^{\circ}, T=\left(27 \pm 0.1^{\circ} \mathrm{C}\right), 0 \mathrm{~dB}, \mathrm{P} \perp \mathrm{A}$. The right curve : ATIR at the interface : $\varphi_{0}=14^{\circ} 14^{\prime}, T=(26 \pm 0.1)^{\circ} \mathrm{C}$, $0 \mathrm{~dB}, \mathrm{P} \perp \mathrm{A}$.

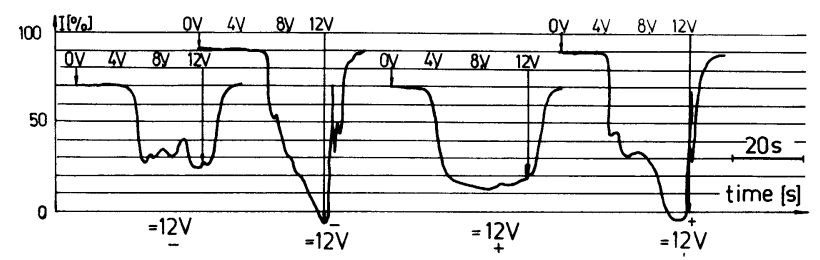

Fig. 11. - Light intensity measurements performed with Brüel and Kjaer recorder, type 2305 , working with a linear potentiometer $10-35 \mathrm{mV}$ in $12 \mu k$ initially deformed MBBA layer. The flint-glass was coated with $\mathrm{Cr}$, the common glass, with $\mathrm{SnO}_{2}$. Narrow curves : light intensity measurements in a transmitted light : $0 \mathrm{~dB}, \mathrm{P} \perp \mathrm{A}$, $T=(29 \pm 0.1){ }^{\circ} \mathrm{C}$. Wider curves-light intensity measurements at ATIR reflected light, $\varphi_{0}=60^{\circ}$.

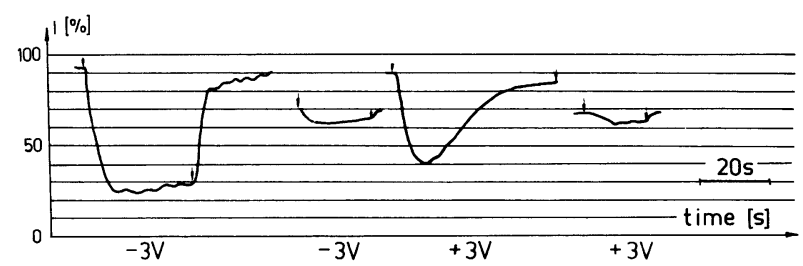

Fig. 12. - Light reflectivity measurements performed with Brüel and Kjaer, type 2305, working with a linear potentiometer 10$35 \mathrm{mV}$ in $12 \mu k$ MBBA layer. The electrodes are from Cr. Small deformations : light intensity measurements at ATIR, $\varphi_{0}=60^{\circ}$, initially homeotropic MBBA layer, $T=(29 \pm 0.1)^{\circ} \mathrm{C}$. Large deformations : initially deformed MBBA layer - the same conditions.

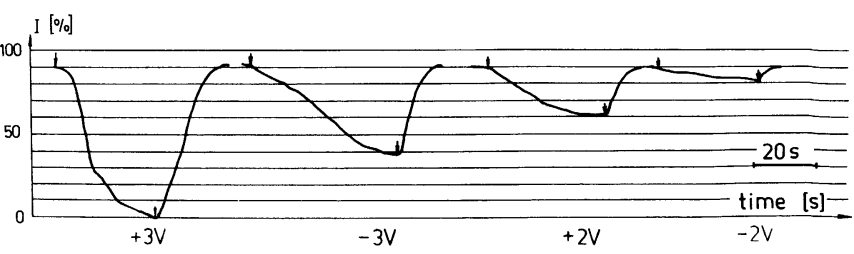

Fig. 13. - Light reflectivity measurements performed with Brüel and Kjaer, type 2305, working with a linear potentiometer $10-35 \mathrm{mV}$ in $45 \mu k$ initially deformed MBBA layer. The flint-glass was coated with $\mathrm{Cr}$ and the common glass, with $\mathrm{SnO}_{2}, \varphi_{0}=60^{\circ}$, $T=(25 \pm 0.1)^{\circ} \mathrm{C}, P / / A, 10 \mathrm{~dB}$.

Using the theory of Nehring's et al. [23] the authors calculated the theoretical curves for two $\lambda$ parameters, characterizing the strength of the surface coupling 
(Fig. 8 and Fig. 9). The following conclusions can be drawn :

1) In d.c. realignment double electric layers [69] exist in the thin cells, the exact values of the electric fields are not known and a large difference between the d.c. and a.c. experimental curves (Figs. 8 and 9) exists for this reason. ATIR permits the investigation of the surface phenomena including the ion behaviour. ATIR shows that there are considerable processes in the cathode region : when the electric field is applied after some times (tenth of a second to $60 \mathrm{~s}$ ) depending on the amplitude of the electric field and the cell thickness a large deformation is established inside the liquid crystal layer. The comparison between the d.c. and a.c. experimental curves (Figs. 8 and 9) permits the determination of the value of the electric field in the electrode region which is about two times larger than that in the middle of the liquid crystal cells. This fact is in agreement with other results [70]. For thicker cells with thicknesses larger than $30 \mu$ the electric field is more homogeneous (see the curves pointed out in Fig. 9).

2) The comparison between experimental and theoretical results permits the determination of the surface energy in thin $(\sim 12 \mu k)$ homeotropic MBBA layers (Fig. 8) which is close to $2 \times 10^{-2} \mathrm{erg} / \mathrm{cm}^{2}$ above the threshold. The validity of this results in the frame of the approximation (41) was verified in accordance with the more complete condition for ATIR (40) (see the theory). The surface behaviour of thicker cells is more complicated (Fig. 9).

3) The upper curves on figures 8 and 9 show the behaviour of initially realigned liquid crystal layers (without lecithin in the flint-glass-liquid crystal interface). In this case the surface coupling along $\theta$ is strong.

4) The comparison between the curves showing the intensity of the reflected light for d.c. and a.c. electric field excitation (Figs. 10 and 11) shows the existence of a strong gradient flexoelectric effect [70] especially in initially deformed homeotropic nematic layers in asymmetrically performed cells (with $\mathrm{Cr}$ and $\mathrm{SnO}_{2}$ as conducting layers) (Figs. 12 and 13). One can also observe the effect of the impurity ions which contribute to the increase of the electric field non homogeneity and to stronger flexoelectric deformations (Figs. 12 and 13). For qualitative estimate one can use symmetrical nematic cells with electrode from noble metals. The task for ATIR measurements of the flexoelectric behaviour of the nematic liquid crystal including and thermal fluctuations goes out of the frame of this paper and will be published in a separate paper [71].

6. Conclusions. - Known formulae are theoretically verified and used in the ATIR experiment performed in deformed initially homeotropic MBBA layers. They allow for the determination of surface deformation observed at attenuated total internal reflections (ATIR).

The ATIR conditions obtained are valid only for deformed homeotropic layers or for deformed tilted layers when the molecular oritentation goes planar in the middle region of the liquid crystal cells. The ATIR conditions for deformed planar layers or deformed tilted layers when the molecular orientation goes homeotropic in the middle region of the liquid crystal cells are different and more complex and require a separate discussion [72].

The ATIR method is especially useful for homogeneous, tilted and homeotropic layers, for an investigation of periodic deformation along $Z$, for liquid crystal layers with a thicknesses in the range of millimeters when the bulk behaviour is not controlled with the aid of the surface anchoring [73], [74], etc...

One can obtained informations about the liquid crystal deformation in the depth of the cells under investigation, the formation and disappearing of the double layers, the surface polarization, the surface flexoeffect, the behaviour of the surface disclinations and a number of other surface effects.

One disadvantage of this method is the utilization of flint-glass prism which is affected at higher temperatures and consequently the deposition of the conductive semi-transparent layers is difficult. On the other hand the ATIR construction of the liquid crystal cell is not typical for the experiments performed and for the displays utilized.

Further improvements of the experiments e.g. mesurements of light spot intensities under dynamic conditions, measurements of the maximum deformations inside the liquid crystal layers, a more exact determination of the temperature separation of thermal fluctuations from the mean molecular orientation (influencing $n_{\text {eff }}$ ) are desirable.

Acknowledgments. - The authors are grateful to Drs. Kh. Pushkarov and N. Sabotinov and to I. Bivas and M. Mitov for discussions. H. Hinov is indebted to Profs. E. Guyon and J. Billard and to Drs. D. Rivière and Y. Levy for the helpful and useful remarks.

The authors also acknowledge the Revue de Physique Appliquée referee for the critical comments leading to the final version of the paper.

\section{References}

[1] Guyon, E., Pieranski, P. and Boix, M., Lett. Appl. Eng. Sci. 1 (1973) 19.

[2] Urbach, W., Borx, M. and Guyon, E., Appl. Phys. Lett. 25 (1974) 479.
[3] Raynes, E. P., Revue Phys. Appl. 10 (1975) 117.

[4] Meyerhofer, D., Phys. Lett. A 51 (1975) 407.

[5] Baur, C., Witlwer, V. and Berreman, D. W., Phys. Lett. A 56 (1976) 142. 
[6] SCheffer, T. J. and Nehring, J., J. Appl. Phys. 48 (1977) 1783.

[7] Freedericksz, V. and Zwetkoff, V., Phys. Z. Sowjetunien 6 (1940) 490.

[8] Dreher, R. and Meier, G., Mol. Cryst. Liq. Cryst. 13 (1971) 17.

[9] Shtrikman, S. and Tur, M., J. Opt. Soc. Am. 64 (1974) 1178.

[10] Tako, T., Akahane, T. and Masubuchi, S., Japan J. Appl. Phys. Suppl. 14-1 (1975) 425.

[11] Dreher, R. and Meier, G., Solid State Commun. 13 (1973) 607.

[12] Fringeli, U. P., Schadt, M., RihaK, P. and Gunthard, HS. H., Z. Naturforsch. 31a (1976) 1098.

[13] Hatta, A., Bull. Chem. Soc. Japan 50 (1977) 2522.

[14] Hatta, A., Bull. Chem. Soc. Japan 51 (1978) 967.

[15] Kashnow, R. A. and Stein, C. R., Appl. Opt. 12 (1973) 2309.

[16] Labrune, G. and Valette, S., Appl. Opt. 13 (1974) 1802

[17] Rivière, D., Thèse, Centre Universitaire d'Orsay, Paris XI (1978).

[18] Rivière, D., LeVY, Y. and ImBert, C., Opt. Commun. 25 (1978) 206

[19] Levy, Y., Rivière, D., Imber T, C. and Borx, M., Opt. Commun. 26 (1978) 225

[20] Rivière, D., Levy, Y. and Guyon, E., J. Physique Lett. 40 (1979) L-215.

[21] Naemura, SH., Appl. Phys. Lett. 33 (1978) 1.

[22] Brunet-Germain, M., C. R. Hebd. Séan. Acad. Sci. B 271 (1970) 1075.

[23] Nehring, J., Kmetz, A. R. and Scheffer, T. J., J. Appl. Phys. 47 (1976) 85.

[24] Derzhanski, A., Petrov, A. G., Hinov, H. P. and MarkovSKI, B. L., Bulg. J. Phys. 1 (1974) 165.

[25] Prost, J. and Pershan, P. S., J. Appl. Phys. 47 (1976) 2298.

[26] Flournoy, P. A. and Schaffers, W. J., J. Spectros. Acta 22 (1966) 5.

[27] Wunsche, A., Ann. Phys. 32 (1975) 401.

[28] Giarusso, D. P. and Harris, J. H., J. Opt. Soc. Am. 63 (1973) 138.

[29] Vassel, M. O., J. Opt. Soc. Am. 64 (1974) 166.

[30] Chanin, D. J., RCA Rev. 35 (1974) 652.

[31] Hu, CH., WhinNeRY, J. R. and AMER, N. M., IEEE J. Quantum Electron. QE-10 (1972) 218.

[32] Hu, CH. and WhinNeRY, J. R., IEEE J. Quantum Electron. QE-10 (1974) 556.

[33] WhINNERY, J. R., Hu, CH. and KNIw, Y. S., IEEE J. Quantum Electron. QE-13 (1977) 262.

[34] Billard, J., Thesis, University of Paris (1966).

[35] SмITH, D. O., Opt. Acta 12 (1965) 13.

[36] Teitler, S. and Henvis, B. W., J. Opt. Soc. Am. 60 (1970) 830.

[37] Berreman, D. W. and Scheffer, T. J., Phys. Rev. Lett. 25 (1970) 577.

[38] Berreman, D. W., J. Opt. Soc. Am. 62 (1972) 502.

[39] Berreman, D. W., J. Opt. Soc. Am. 63 (1973) 1374.
[40] Shimomura, T., Mada, H. and Kobayashi, SH., Japan J. Appl. Phys. 15 (1976) 1479.

[41] Al'pert, R. L., Ginsburg, V. L. and Feinberg, E. L., Radiowaves propagation, Moscow 1953.

[42] LANDAU, L. D. and LiFshitZ, E. M., Electrohydrodynamics of continuous media (Pergamon Press) 1960.

[43] Brekhovskikh, L. M., Waves in layered media (Academic Press) 1960.

[44] Jefrereys, H. and Jefrreys, B. S., Methods of Mathematical Physics (Cambridge University Press) 1946.

[45] Olver, F. W. J., Philos. Trans. R. Soc. London Ser. A 247 (1954) 307.

[46] Smirnov, V. I., High Mathematical Course, III pt. II, p. 431, Moscow (1974).

[47] KамкE, E., Differential Equations, Leipzig, 1959.

[48] Mahan, A. I. and Bitterli, C. V., Appl. Opt. 17 (1978) 509.

[49] Hinov, H. P., III LC conference of the soc. countries, Budapest (1979) B 19.

[50] Mada, H. and Kobayashi, SH., Mol. Cryst. Liq. Cryst. 33 (1976) 47.

[51] These experimental results were reported in a preliminary form in 1974 : Hinov, H. P. and SaINov, S., VI Nat. Conf. on Spect., Slanchev Bryag, Bulgaria, Sept. 30Oct. 3, Abstracts N 9, 248.

[52] HARRICK, N. J., Internal Reflection Spectroscopy (Interscience Pub. John Wiley \& Sons) ch. IV, 1967.

[53] Green, M., Kirkby, P. and Timsit, R. S., Phys. Lett. 45A (1973) 259.

[54] Kozaki, S. and Mushiake, Y., J. Appl. Phys. 46 (1975) 4098.

[55] Kozaki, S. and Sakurai, H., J. Opt. Soc. Am. 68 (1978) 508.

[56] Kozaki, S. and Harada, H., J. Opt. Soc. Am. 68 (1978) 1592.

[57] Montgomery, G. P., Res. Pub. GMR-3033 (1979) 1.

[58] Montgomery, G. P., Res. Pub. GMR-3043 (1979) 1.

[59] Montgomery, G. P., Res. Pub. GMR-3055 (1979) 1.

[60] De Gennes, P. G., Mol. Cryst. Liq. Cryst. 7 (1969) 325.

[61] Orsay Group on liQuid CRYSTALS, J. Chem. Phys. 51 (1969) 816.

[62] Langevin, D., Bouchiat, M. A., J. Physique Colloq. 36 (1975) C1-197.

[63] Leger, L., Thèse de 3e Cycle, Université de Paris XI (1970).

[64] Hu, CH. and Whinnery, J. R., J. Opt. Soc. Am. 64 (1974) 1424.

[65] Giallorenzi, T. G. and Sheridan, J. P., J. Appl. Phys. 46 (1975) 1271

[66] Hinov, H. P., VII Int. Conf. on LCs., Bordeaux, France (1978) HP-55.

[67] Chang, R., Mol. Cryst. Liq. Cryst. 28 (1974) 1.

[68] Labrunie, G. and Robert, J., J. Appl. Phys. 44 (1973) 4869.

[69] Sprokel, G. J., Mol. Cryst. Liq. Cryst. 22 (1973) 249.

[70] Derzhanski, A. I., D. Sci. Thesis (1979) Sofia.

[71] Hinov, H. P., to be published.

[72] GuYon, E., private communication.

[73] Hinov, H. P., submitted for publication in J. Physique.

[74] Tsvetkov, V. N., Ph. D. Thesis (1974) Moscow. 